\title{
Јитература
}

1. [http://www.pasterizator.ru/art2.htm]

2. [http://www.ecomash.ru/publication/22.html]

\section{АВТОМАТИЗИРОВАННОЕ ПРОЕКТИРОВАНИЕ ТЕХНОЛОГИЧЕСКИХ ПРОЦЕССОВ С ДИСКРЕТНЫМ ХАРАКТЕРОМ ПРОИЗВОДСТВА}

\author{
Водяницкая А. А., Гороховатская Н. В. \\ научный руководитель к. Т. н. проф. Кузьменко В. М. \\ Харьковский национальный университет радиоэлектроники, Г. Харьков
}

В работе рассматривается проблема автоматизаиии проектирования структур технологических прочессов с дискретныи характером производства. В работе разработан эвристический метод синтеза структуры на основе усеченного поиска в глубину. Предложенный иетод решения проблемы реализован в виде алгорития, на основе которого разработано автоматизированное средство проектирования.

In this work the problem of synthesis of industrial structure for processing of discrete material streams is considered. Statement of a problem of industrial structure design, and also the generalised algorithm of the decision of this problem is developed.

Ключевые слова: технологический процесс, дискретный материальный поток, информационные технологии, математическая модель, структура, программное средство, программная документация, надежность.

Введение. Ни одно производственное предприятие не обходится без технологических процессов. Технологический процесс (ТП) может протекать, как в рамках одного отдела, так и в масштабах всего предприятия. Существует множество научных трудов, посвященных видам ТП, их обслуживанию, оптимизации, созданию требований, а также многих других. Тем не менее, разработка подходов к синтезу физической структуры ТП сегодня актуальна как никогда. Это объясняется автоматизацией производства в целом и технологических процессов в частности. Создание целых линий, состоящих из механических машин, предполагает детальное проектирование и синтез производственных структур на основе особенностей одного или группы технологических процессов.

Таким образом, задача проектирования структуры ТІІ состоит в изучении существуюцих технологий, оборудования и его производственных характеристик, получении непосредственно набора структур, позволяющих преобразовать некоторый материальный поток, который поступает на вход структуры, а также в анализе полученного набора структур. Этап анализа необходим для выбора из множества одной структуры, удовлетворяющей требованиям данного ТП. Особенности технологического процесса можно учитывать с помощью ограничений, четко сформулированных и представленных в математической форме. Также необходимо выделить основные критерии анализа структур, то есть необходимо заранее определить параметры выбора наиболее подходящей структуры. Чтобы формализовать постановку задачи, предлагается выделить целевую функцию, с заданными критериями оптимизации, а входные параметры ТП и его ограничения записать с помощью множеств.

Целевая функция имеет вид:

$$
\mathrm{C}=\min \left(\mathrm{Z}, \mathrm{T}_{\text {обр }}\right)
$$

Параметрами целевой функции являются: $Z$ - общие материальные затраты на создание и обслуживание ТП; $T_{\text {обр }}$ - суммарное время, затрачиваемое на обработку потока заданного объема.

Входные данные: входящий материальный поток $V$; множество оборудования различных видов $\left\{\mathrm{R}^{*}\right\}$; набор технологий, то есть множество технологических операций $i(i=\overline{1, n})$ и связей между видами оборудования на разных операциях $\left\{\mathrm{Tx}^{*}\right\}$; производительность оборудования $\left\{\mathrm{U}^{*}\right\}$; начальная стоимость оборудования $\{\mathrm{Zn}\}$; эксплуатационная стоимость $\{\mathrm{Ze}\}$.

Ограничения: на заданный состав оборудования $R \leq R^{*}$; на заданный набор технологий $T x \leq T x^{\circ}$; на производительность оборудования $U \leq U^{*}$; на площадь производственного участка $S \leq S^{*}$. 
Технологический процесс - часть производственного процесса, содержащая целенаправленные действия по изменению и /или определению состояния предмета труда. Приведенная выше постановка задачи описывает технологические процессы в течение которых обрабатываемые объекты не меняют своей материальной формы. К предприятиям подобного типа можно отнести предшриятия и организации нешромышленной среды: фирмы, выполняющие оптовую и розничную продажу товаров, складские предприятия, сеть почтовой связи, завершаюших подразделений производственных предприятий, экспедиционных предприятий и др. [3].

На данный момент мелкие предприятия решают проблему проектирования структуры ТІІ либо за счет опыта, наработанного лицом принимающим решение (ЛІР), либо за счет ручного расчета вариантов использования того или иного вида оборудования на каждой гехнологической операции. Также для обработки документооборота и осуществления экспедирования используется транспортная и информационная логистика, однако ни один из перечисленных подходов не решает проблемы синтеза структуры ТП.

Рассматривать технологический процесс удобнее с точки зрения разбиения на технологические операции. Технологический процесс обработки любого вида отправлений представляет собой совокупность отдельных элементов - технологических операций. Понятие «технологическая операция» впервые появилось с развитием мануфактурного производства путем разбиения общего объема обработки на отдельные взаимосвязанные части, которые выполнялись на отдельных рабочих местах и требовали применения груда работников различной профессиональной подготовки. Сложившееся определение технологической операции остается справедливым и для таких технологических процессов, основным участником которых является человек, что составляет существенную долю в общем производственном процессе на предприятиях. Отсюда технологическая операция - это однородная составляюцая производственного процесса, выполняемая на определенном рабочем месте одним или несколькими работниками [2]. В условиях автоматизированного производственного процесса технологическая операция с одной стороны становится частью рабочего инкла машины, а с другой стороны для функционально сложных операций может быть использована технологичцская линия, состоящая из нескольких машин и механизмов. Живой человеческий труд в данном случае применим, как правило, для организации «гибких» связей между отдельными машинами и механизмами, которые в настоящий момент по тем или иным причинам не могут или не подлежат автоматизации [2]

Метод решения. Задача сводится к графу вершинами которого являются альтернативы оборудовыния на каждой технологической операции, а дуги показывают связи между альтернативами на разных операциях. Для минимизации функции цели необходимо найти путь, минимальный по затратам $Z$ и по времени обработки $\mathrm{T}_{\text {обр }}$. Для преобразования многокритериальной задачи в однокритериальную время обработки потока переведем в ограничения.
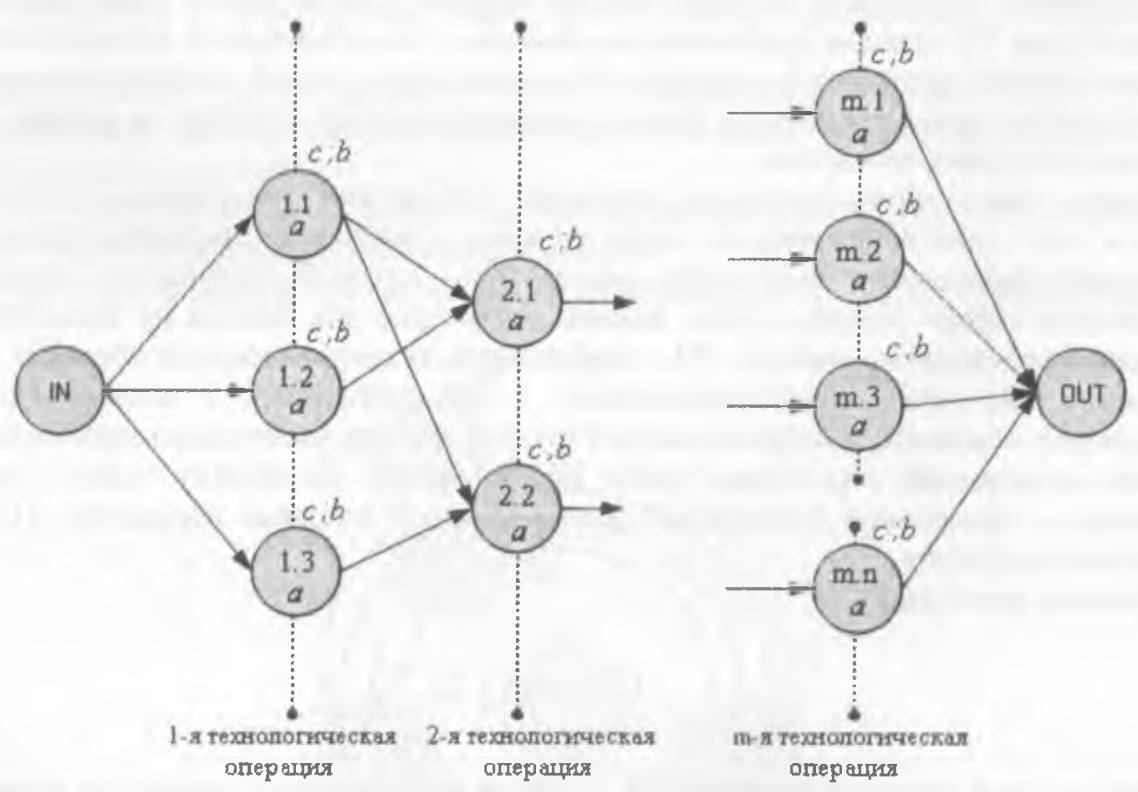

Рис. 1 - Графическая модель ТП

ІІолучаем неклассическую задачу поиска пути с наименьшей стоимостью и ограничением по времени на графе, с изменяемым коэффициентом для каждой вершины (т е изменяется количество одного вида оборудования на операции). Классических методов принятия решений для этой задачи нет, поэтому был предложен алгоритм решения с помоцью модифицированного поиска вглубину с оценкой времени (рис. ).

Суть метода заключается в усеченном переборе вершин с помощьо рекурсивного алгоритма. С его помоцью мы вычисляем стоимость применения данного вида оборудования на данной операции с учетом уже пройденного пути и время обработки заданного входящего потока. Далее сравниваем полученные значения 
времени с ограничением, если оно удовлетворяет ограничениям, сравнивасм полученную стоимость с минимальной. Метод не рассматривает дальше начатый путь, если стоимость больше предыдущей минимальной найденной, а также если время обработки не удовлетворяет ограничению (в этом состоит усечение перебора). Таким образом рассматриваются только «перспективные» пути, при чем «перспективность» оценивается на каждом шаге. Если вершина нас устраивает, переходим к перебору по количеству данного вида оборудования, т е масштабирует вершину. ІІри каждом следующем изменении количества оборудования, алгоритм рекурсивно вызывает сам себя и пересчитывает значения времени обработки и стоимости. IІосле определения оптимального количества альтернативы, ищем связь с вершинами на следующей операции. Если такие имеются, описанная процедура рекурсивно повторяется для каждой вершины, с которой есть связь.

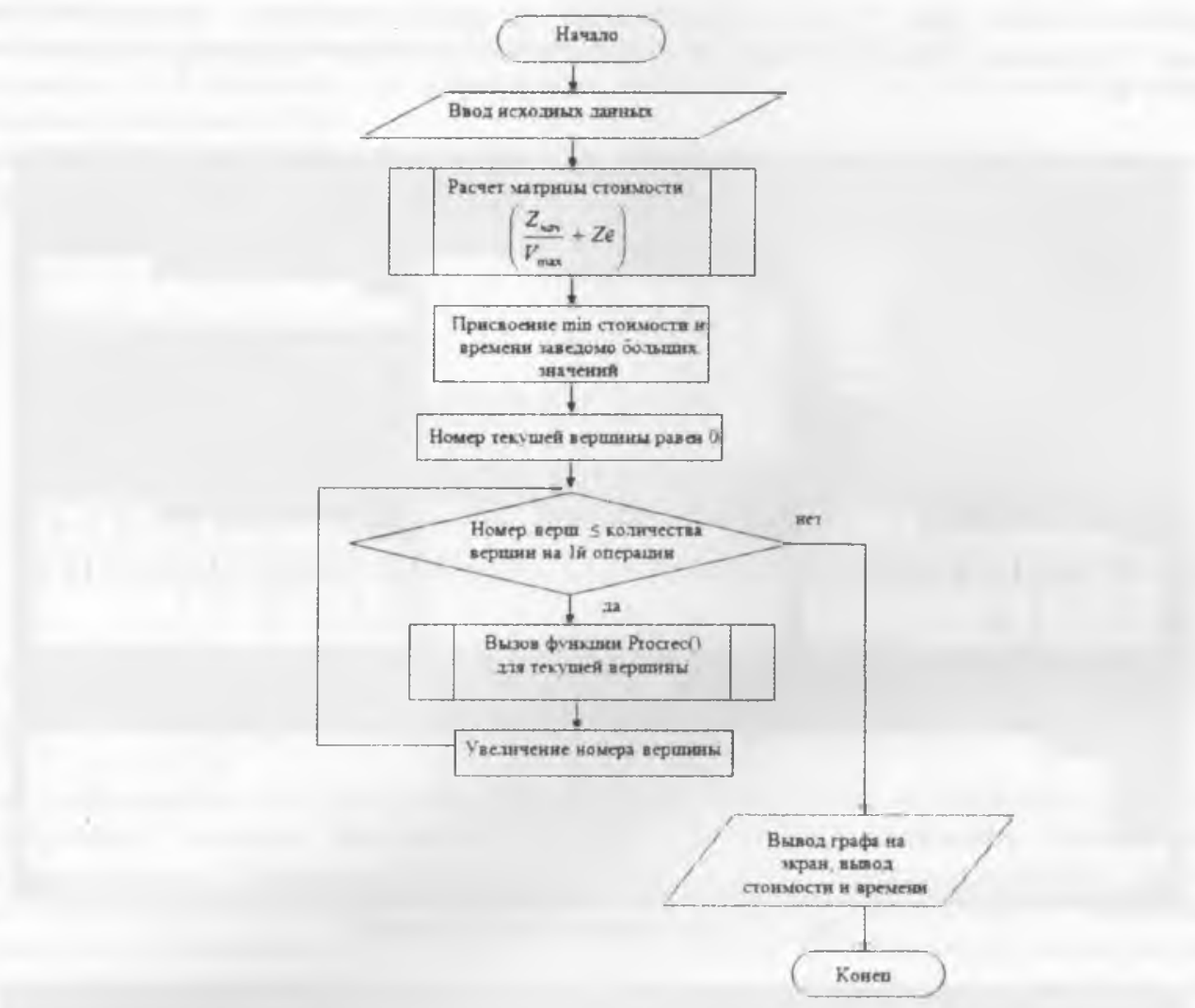

Рис. 2 - Схема обоб̋енного алгоритма проектирования структуры ТП

На каждом шаге кроме расчета стоимости для каждой вершины необходимо рассчитывать и время обработки. На длительность производственного цикла существенно влияет способ выполнения производственных операций. Различают три основных способа выполнения производственных операций последовательный, параллельный и параллельно-последовательный. Наиболее эффективным с точки зрения загрузки оборудования является параллельно-последовательный способ. Это способ выполнения производственных операций, когда начало выполнения каждой последуюшей операции смещено во времени но отношению к началу выполнения каждой предшествующей. На рис.3 приведена диаграмма Ганта, которая показывает время обработки входящего объема на каждой технологической операции.

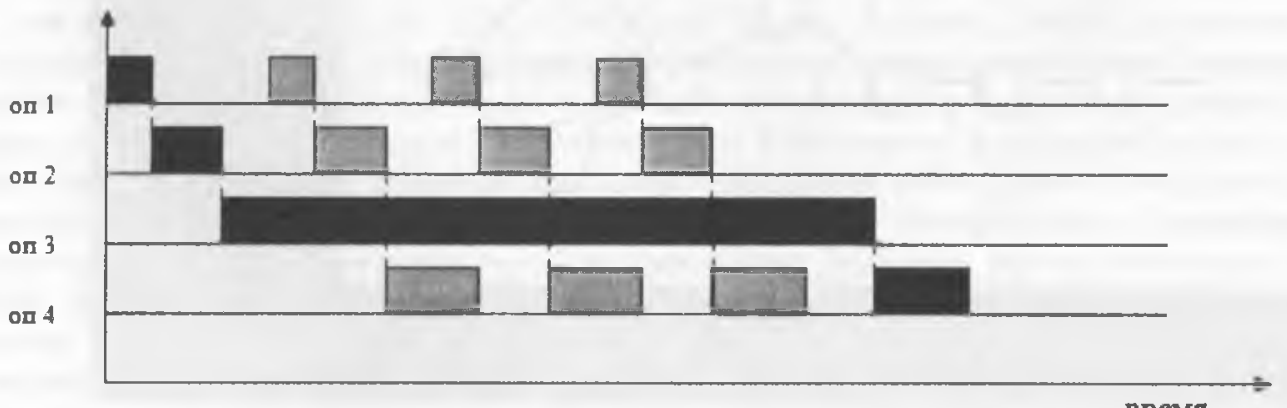

Рис. 3 - Диаграмма Ганта 


$$
T_{\text {par }}=P \sum_{i=1}^{m}\left(\frac{t_{i}}{c}\right)+(n-p)\left(\frac{t_{i}}{c}\right)_{\text {long }},
$$

где C - число агрегатов, выполняющих одинаковые функции; P - передаточная партия; Т - время обработки всей партии объемом $\mathrm{n} ; \mathrm{n}$ - объем партии; $\mathrm{m}$ - число операций в данном процессе; с - время обработки единицы входяцего потока; long - самая длинная операция.

Реализация. Предложенный метод реализован в программе StructureDesign_v4_0 (рис 4). Данное программное средство (ПС) предназначено для проектирования структуры дискретных технологических процессов на основе данных об оборудовании, технологиях обработки объектов входящего материального потока и стоимости обработки. ПС позволяет рассчитать стоимость применения каждого вида оборудования на конкретной операции ТП, сформировать структуру TII, которая обеспечивает минимальную стоимость обработки входящего потока (рис. 5) при ограничениях на время обработки, представить структуру в графическом виде. I Ірограмма StructureDesign_v4_0 разработана на языке программирования C ++ в среде Borland Builder C ++6 .

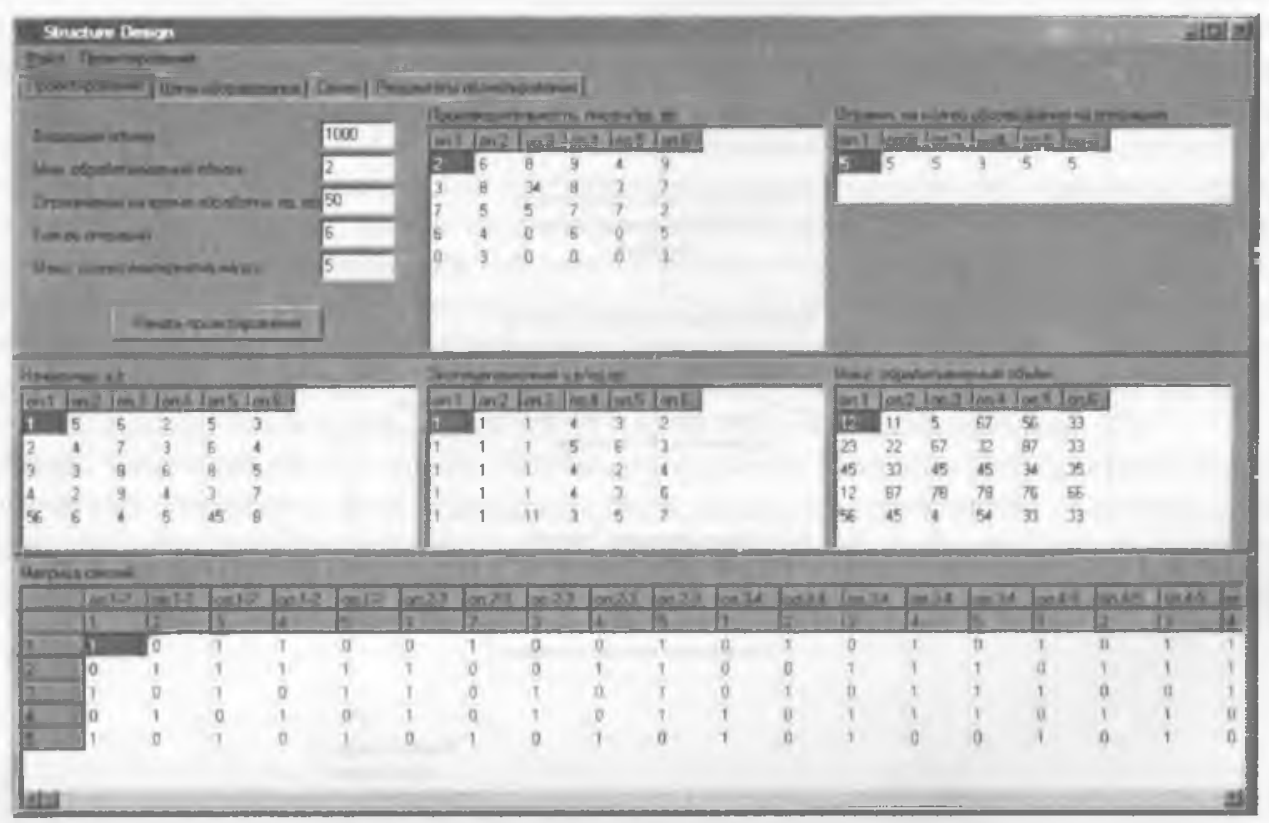

Рис. 4 - Интерфейс ввода данных

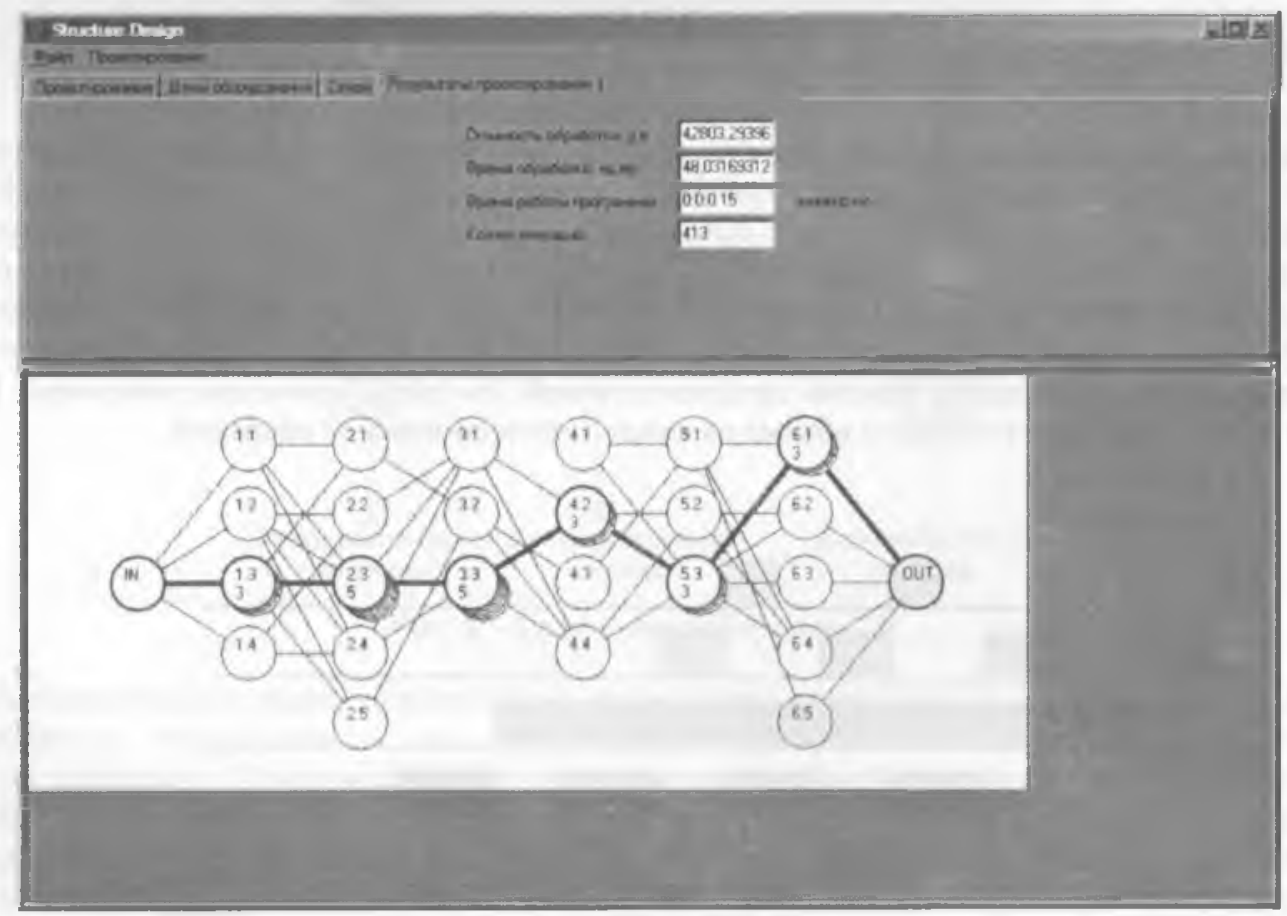

Рис. 5 - Результаты работы программы 
Выводы. В ходе решения задачи проектирования структуры ТІІ был выполнен анализ, сформулирована постановка задачи проектирования производственной структуры, предложен эвристический метод решения и реализован в виде программы. Разработанный метод дает точный результат и может быть использован для технологических процессов, имеющих не более десяти операций, гак как при их увеличении трудоемкость алгоритма резко возрастает. Использование данного программного средства позволяет оператору решить задачу определения оптимального состава оборудования и тем, самым повысить эффективность деятельности предприятия.

В дальнейшем необходимо: модифицировать метод для обработки непрерывного материального потока; решить задачу последовательности запуска материалов на обработку; синтезировать имитационную модель структуры технологического процесса и провести имитационныс эксперименты.

\title{
Јитература
}

1. Глазунов В.Н. Поиск принципов действия технических систем М: 1990.

2. Кандырин Ю.В., Шкрина Г.Л. Процедуры генерации и выбора при проектировании технических объектов Волгоград, 1999.

3. Организация производственных процессов на механизированных почтовых предприятиях / И. И. Жадько, С. Н. Скляренко, Я. М. Корж. - . - Москва : Связь, 1979. - . 222 с.

\section{ПОВЫШЕНИЕ ЭНЕРГЕТИЧЕСКОЙ ЭФФЕКТИВНОСТИ ТЕПЛОВЫХ ПРОЦЕССОВ ПИЩЕВЫХ ТЕХНОЛОГИЙ СРЕДСТВАМИ ГАРАНТИРУЮЩЕГО УПРАВЛЕНИЯ: УПРАВЛЕНИЕ ПРОЦЕССОМ ПАСТЕРИЗАЦИИ МОЛОКА}

\author{
Хоб̈ин В.А., Д-р техн. наук, профессор, Мазур А.В., канд. техн. наук, цоцент, \\ Стенанов М.Т., канд. техн. наук, доцент \\ Одесская национальная академия пищевых технологий, г. Одесса
}

\begin{abstract}
В статье рассматривается путь снижсени энергетических затрат на ведение технологических процессов тепловой обработки пицевых продуктов, связанный с совериенствованиеи систем автоиатического управления прочессами. Сформулированы два приниипа такого совериенствования. Первый состоит в повыиении динамической точности стабилизации регулируеиых температур в окрестности заданных значений. Второй - в изменении этих заданных значений таким образом, чтобы температуры иаксимально приближались $k$ их предельно допустимым значениям, не наруиая их с заданной гарантированной вероятностью. Эффективность сформулированных принципов иллюстрируется на примере пастеризации молока.

The questions of energy expenses reduction in technological processes of thermal processing foodstuff due to perfection of automatic control systems are considered. Two principles of such perfection are formulated. The first consists in increase of dynamic accuracy of stabilization of regulated temperatures of process in a vicinity of preset meaning. The second - in change of these given meaning so that these temperatures as much as possible approach to the own maximum-permissible meaning, but with beforehand guaranteed probability did not leave outside them. On the example of work pasteurizing plant the essence of offered principles is illustrated, and results of their application are shown.
\end{abstract}

Ключевые слова: пишевые технологии, тепловая обработка, автоматическое управление, энергосбережение, качество.

Технологические процессы (TII), движущей силой которых является разность температур сред, участвующих в процессе, а целью взаимодействия этих сред является изменение температуры одной из них, называют тепловыми. В отраслях пищевой промышленности это, прежде всего, процессы тепловой обработки, которые составляют значительную часть технологий производства и хранения пицевых продуктов. Являясь весьма энергоемкими, они в значительной степени определяют себестоимость продукции. Стремление снизить энергетическую составляющую затрат в свое время стимулировалю разработку и применение энергосберегающего оборудования, предусматривающего различныс формы утилизации тепла. К настоящему времени ресурсы энергосбережения за счет утилизации тепла практически исчерпаны. Необходимы новые nодxоды. Не отрицая большой значимости в этих подходах поиска принципиально новых технологий, подчеркнем, что традиционные технологии сегодня реализуются энергетически недостаточно эффективно Термин «реализуются» указывает на то, что в значительной мере эта неэффективность обуславливается неэффективностью управления этими теІловыми TII (TTII). Важно отметить, что когда идет речь об 